\title{
TERAPIA ENDOVESICAL: INFLUENCIA SOBRE LA RECIDIVA EN EL CÁNCER VESICAL SUPERFICIAL.
}

Jorge García Rodríguez, Jesús María Fernández Gómez, Safwan Escaf Barmadah, Roberto Carlos González Alvarez, Antonio Jalón Monzón, Francisco Javier Martínez Gómez, Alberto Sánchez Trilla y Javier Regadera Sejas.

Servicio de Urología I. Hospital Central de Asturias. Oviedo. Asturias. España.

Resumen.- OBJETIVO: Revisar la influencia de distintos tratamientos endovesicales sobre la recurrencia del cáncer vesical superficial.

MÉTODOS: Se revisaron retrospectivamente un total de 473 neoplasias vesicales superficiales. A partir del diagnóstico y la RTU vesical, y en función del resultado anatomopatológico, se plantearon distintos esquemas de seguimiento y tratamiento complementario.

RESULTADO: Resultaron independientes para explicar mayor tiempo libre hasta la recurrencia superficial los siguientes factores: empleo de lavados endovesicales, tratamiento con dosis altas de BCG y terapia de mantenimiento frente a inducción.
Jorge García Rodríguez Martínez Vigil, 2 - 2ํ dcha 33010 Oviedo. (España) igrmed@hotmail.com Trabajo recibido: 18 de junio 2006
CONCLUSIONES: El empleo de instilaciones vesicales incrementaron de forma independiente el tiempo hasta la recidiva. Se demostró que la recurrencia se produjo significativamente más tarde cuando se habían empleado dosis altas de BCG y terapia de mantenimiento.

Palabras clave: Cáncer vesical superficial. Recidiva. Tratamiento endovesical.

Summary.- OBJECTIVES: To review the influence of various intravesical treatments on superficial bladder cancer recurrence.

METHODS: We retrospectively reviewed 473 superficial bladder neoplasias. Based on diagnosis and transurethral resection of bladder fumor (TUR BT), and depending on pathology, we proposed different adjuvant treatment and follow-up schemes.

RESULTS: The following factors were independent risk factors for a longer recurrence-free time: intravesical instillations, high dose BCG, and maintenance therapy in comparison to induction.

CONCLUSIONS: The use of intravesical instillations independently increases the time to recurrence. It was demonstrated that recurrence was significantly later when high dose BCG and maintenance therapy were employed.

Keywords: Superficial bladder cancer. Progression. Intravesical treatment. 


\section{INTRODUCCIÓN}

El tratamiento estándar en el cáncer vesical superficial es la resección transuretral (RTU) que, es la única técnica disponible con potencial curativo real (1). Tras el tratamiento endoscópico, según determinados factores pronóstico más o menos favorables, se plantea la posibilidad de aplicar tratamientos adyuvantes endovesicales, frente al riesgo de recurrencia o progresión de la enfermedad. $(2,3)$. Terapias ablativas, por un lado, indicadas en el Cis, tumores múltiples, grados intermedios altos o muy recidivantes y terapias profilácticas, que intentan retrasar la aparición de recidiva o progresión.

Hemos querido revisar la influencia de distintos tratamientos endovesicales en la recurrencia del cáncer vesical superficial. Fundamentalmente, la terapia endovesical con Mitomicina $C(M M C)$, un antibiótico con actividad quimioterápica que actúa inhibiendo la síntesis de ADN, y la inmunoterapia con BCG (Bacilo de Calmette-Guerin), utilizados ambos en forma de instilaciones vesicales según distintos protocolos.

\section{MATERIAL Y MÉTODOS}

Se revisaron retrospectivamente un total de 473 neoplasias vesicales superficiales, de las cuales finalmente 419 fueron útiles para el estudio pues disponían de todos los datos que se querían analizar.

A partir del diagnóstico y la RTU vesical, y en función del resultado anatomopatológico, se plantearon distintos esquemas de seguimiento y tratamiento complementario. En 219 casos (52,3\%) se empleó un tratamiento endovesical tras la RTU del tumor vesical. La inmunoterapia con BCG (La cepa de BCG que utilizamos fue la Connaught), representó un $63,5 \%$ (n: 139) de todos los tratamientos (empleada en el $33,2 \%$ del total de pacientes), utilizando alta dosis $(81 \mathrm{mg}$ ) en 87 casos y dosis más bajas en 52 casos. Se empleó quimioterapia endovesical en 80 pacientes $(19,1 \%$ del total), lo cual representa un $36,5 \%$ de las terapias endovesicales, siendo fundamentalmente Mitomicina C (MMC) (n: 53). Se emplearon regímenes de mantenimiento (más de 6 instilaciones) en 140 casos, tanto inmuno $(65,5 \%$ de los tratados con BCG) como quimioterapia (61,3 de los tratados con quimioterapia), lo cual supone globalmente un $63,9 \%$ de los pacientes que recibieron tratamiento endovesical post-RTU (Tabla I).

El análisis estadístico se realizó mediante el programa SPSS, versión 11 . Se consideró la existencia de significación estadística cuando la $p$ fue menor de 0,05.

\section{RESULTADOS}

No encontramos diferencias significativas cuando analizamos el tamaño medio (t de Student) o multiplicidad tumoral (Chi2) en los pacientes en los que se empleó o no tratamiento endovesical. Sin embargo, observamos que existía una asociación significativa entre el empleo de lavados y el estadio o grado, respectivamente. De esta manera, el empleo de terapia endovesical fue más frecuente en tumores T1 y en los de alto grado (G3 y/o Tis). Un 30\% de tumores Ta recibieron tratamiento endovesical tras RTU frente a un $57,3 \%$ de $\mathrm{T} 1$. Encontramos que el $86 \%$ de tumores de alto grado (G3 y/o Tis) habían recibido lavados tras la RTU vesical, frente a un $50 \%$ de tumores de grado II y un $32,5 \%$ de grado I. Entre los pacientes que recibieron terapia endovesical (n: 219) analizamos el tipo de tratamiento, demostrando que la proporción de pacientes que se trataban

TABLA I. PACIENTES QUE RECIBIERON TERAPIA ENDOVESICAL, DETALLANDO EL TIPO DE LAVADOS IBCG O QUIMIOTERAPIA) Y LA DURACIÓN (INDUCCIÓN O MANTENIMIENTO).

\begin{tabular}{|l|c|c|c|}
\hline & \multicolumn{2}{|c|}{ TIPO LAVADOS } & \\
\hline & INDUCCIÓN & MANTENIMIENTO & Total \\
\hline BCG & 48 & 91 & 139 \\
\hline Quimio & 31 & 49 & 80 \\
\hline Total & 79 & 140 & 219 \\
\hline
\end{tabular}




\section{TABLA II. DISTRIBUCIÓN DE TUMORES SEGÚN EL GRADO Y LA TERAPIA ENDOVESICAL EMPLEADA: QUIMIOTERAPIA; BCG ALTA DOSIS 181 MG CEPA CONNAUGHT) O BAJA DOSIS (MENOS DE 81 MG CEPA CONNAUGHT).}

\begin{tabular}{|l|c|c|c|c|}
\hline & GI & GII & GIII y/o Tis & Total \\
\hline Quimioterapia & 29 & 39 & 12 & 80 \\
\hline BCG baja dosis & 13 & 27 & 12 & 52 \\
\hline BCG alta dosis & 8 & 17 & 62 & 87 \\
\hline Total & 50 & 83 & 86 & 219 \\
\hline
\end{tabular}

con BCG o quimioterapia se asoció estadísticamente al estadio y grado tumoral, pero no al tamaño ni a la multiplicidad. Así, la probabilidad de recibir BCG era 3,5 veces superior a la de recibir quimioterapia en tumores Ta-1G3 y/o Tis (aislado o asociado a un tumor papilar). Sin embargo, esta probabilidad se invertía a favor de quimioterapia en los tumores TIG 1-2 (1,8 veces mayor de recibir quimioterapia) y en las neoplasias TaG 1-2 (2,5 veces mayor de recibir quimioterapia) que recibieron terapia endovesical. En los tumores Ta y $\mathrm{Tl}$ de grados bajo o medio sin Tis empleamos más frecuentemente BCG que quimioterapia cuando fueron múltiples o grandes (no hubo tumores TaG1-2 mayores de $3 \mathrm{cms}$ ), pero no se encontró una asociación estadística en estos tumores entre tamaño o multiplicidad y el tipo de tratamiento (BCG o quimioterapia).

Un 72, 1\% (n: 62) de los pacientes con tumores de alto grado (Ta-1G3 y/o Tis) recibieron inmunoterapia con dosis altas de BCG en cada instilación $(81 \mathrm{mg}$ ), frente a un $20,2 \%$ (n: 24 ) de tumores TIG12. Empleamos dosis bajas de BCG en el $14 \%$ de tumores de alto grado, porcentaje similar al del empleo de quimioterapia. Se utilizaron dosis bajas de BCG en el $30-35 \%$ de tumores grado I y II, porcentaje inferior al empleo de quimioterapia en ambos grupos (Tabla II).

La posibilidad de recibir tratamiento de mantenimiento fue 1,3 veces superior en caso de multiplicidad. Sin embargo, no encontramos asociación entre el tipo de tratamiento (inducción frente a mantenimiento) y el estadio, grado o tamaño tumoral. De esta forma, un $63,4 \%$ de los tumores de alto grado (T1G3 y/oTis) recibieron terapia endovesical de mantenimiento, proporción similar a la de los tumores Ta y Tl G1-2 sin Tis.
Se comprobó que el empleo de instilaciones vesicales incrementó el tiempo hasta la recurrencia, existiendo diferencias estadísticamente significativas (p: 0,001), a favor de los pacientes que recibieron terapia endovesical. La mediana del tiempo libre de enfermedad en los pacientes que no recibieron tratamiento fue de 18 meses (intervalo de confianza del $95 \%$ entre 11-25 meses) y el de los que recibieron tratamiento fue de 37 meses, con un intervalo de confianza del $95 \%$ de 26 a 48 meses (Figura 1).

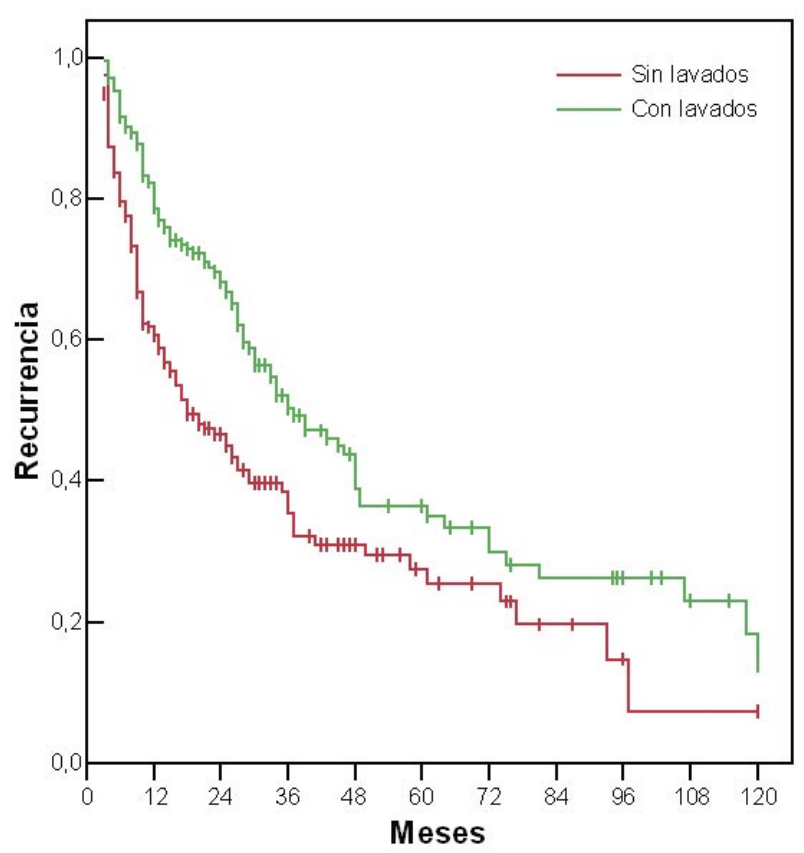

FIGURA 1. Tiempo hasta la recurrencia según se emplearan o no lavados vesicales tras la RTU, mostrando diferencias significativas (p: 0,000 1). 


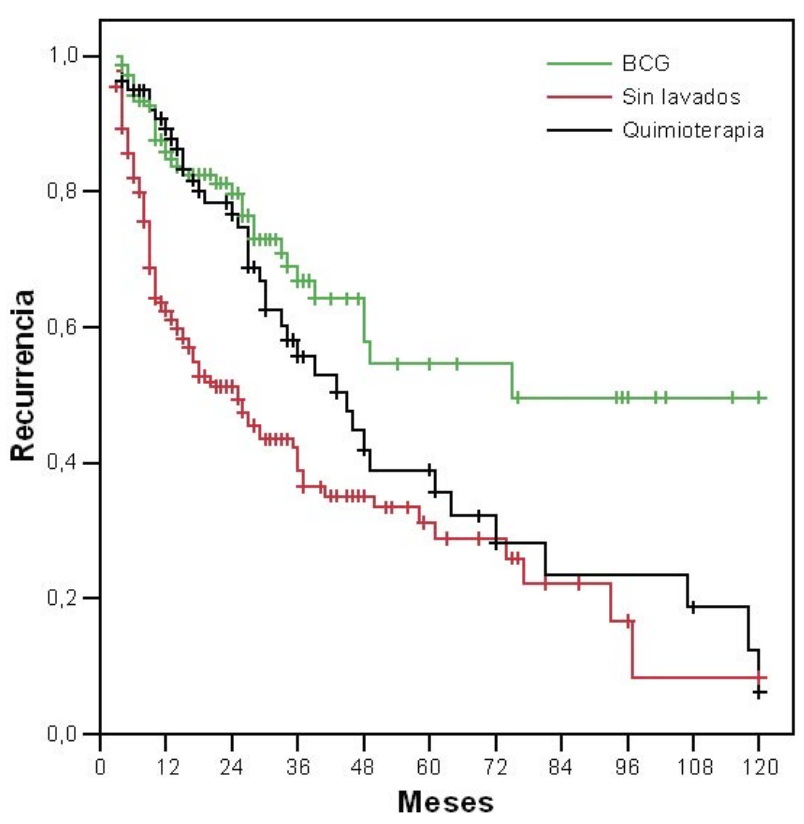

FIGURA 2. Tiempo hasta la recurrencia según se emplearan BCG, quimioterapia endovesical o no se usaran lavados, mostrando diferencias significativas (p $<0,00011$.

En los pacientes en los que se emplearon lavados se demostró que existían diferencias significativas en el tiempo hasta la recurrencia al emplear BCG o quimioterapia con diferentes drogas, fundamental- mente MMC. La mediana en los que fueron tratados con BCG fue de 75 meses, la de los pacientes que habían recibido quimioterapia endovesical fue de 45 meses con un intervalo de confianza del $95 \%$ entre 32 y 58 meses y la de los que no recibieron tratamiento fue de 25 meses (intervalo de confianza del $95 \%$ de 18 a 32 meses) (Figura 2).

En lo referente a las dosis empleadas de $B C G$, se comprobó que existían diferencias significativas en el tiempo hasta la recurrencia (p: 0,0292), con un tiempo mayor en los pacientes que habían recibido dosis altas de $B C G$, frente a los que recibieron dosis bajas de BCG y a los que recibieron quimioterapia. Las curvas de supervivencia de pacientes sin tratamiento fueron superponibles a las de aquellos que habían recibido quimioterapia o dosis bajas de BCG. Se reagruparon en dos grupos para el estudio multivariante, uno de ellos el de BCG a dosis altas y el otro una combinación de tratamiento con BCG de baja dosis quimioterapia o nada, comprobando nuevamente que existían diferencias estadísticamente significativas $(p<0,001)$ en el tiempo medio libre de enfermedad a favor del grupo de dosis altas de BCG (log rank: 23,73) (Figuras 3A y 3B).

Al estudiar la duración del tratamiento endovesical, se demostró que existían diferencias estadísticamente significativas (p: 0,035) cuando compara-
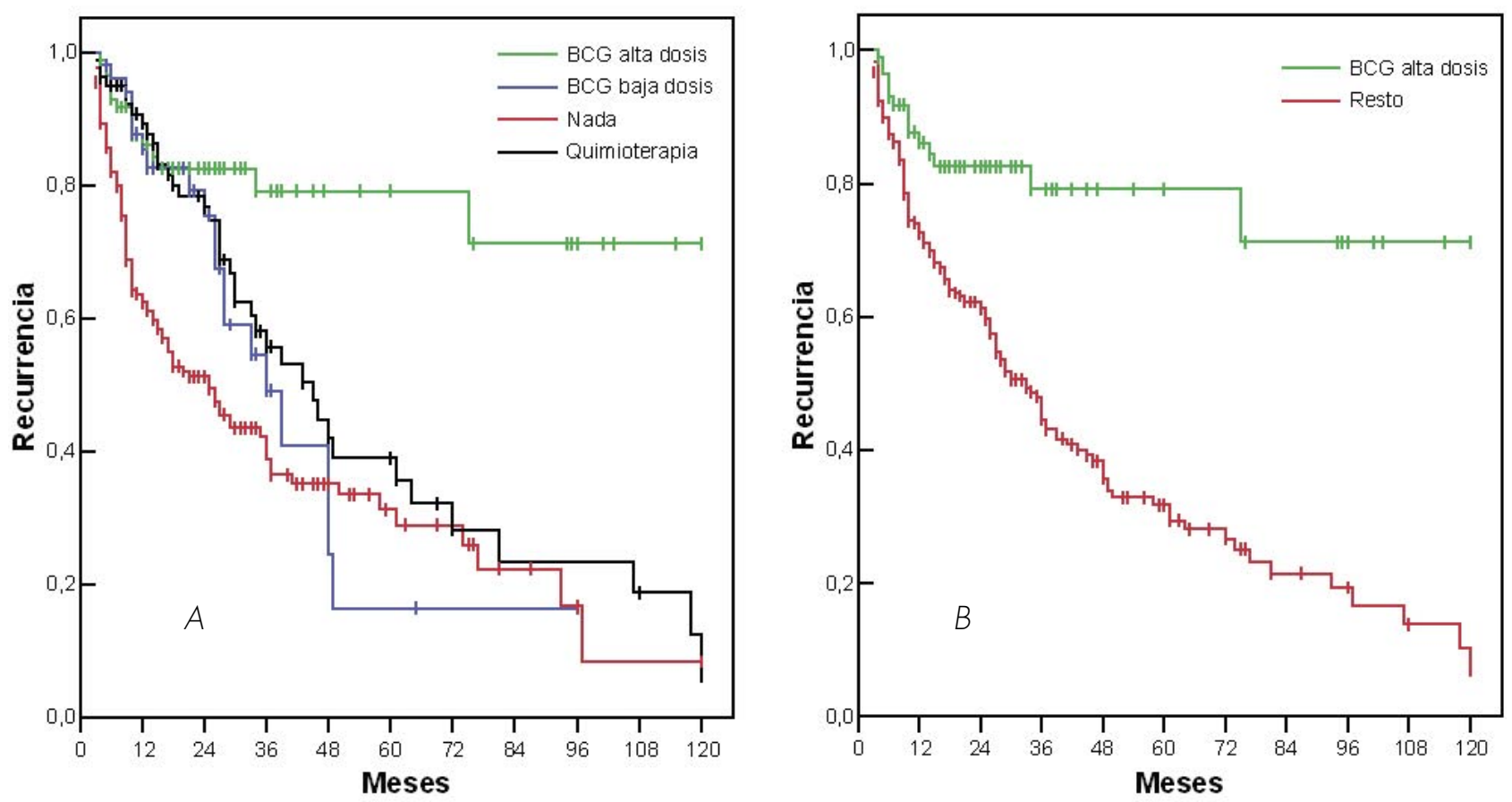

FIGURA 3. Tiempo hasta la recurrencia según se emplearan BCG a alta o baja dosis, quimioterapia endovesical o no se usaran lavados, mostrando diferencias significativas ( $p<0,0001)(3 \mathrm{~A})$. Se reagruparon en casos que recibieron dosis altas de BCG y el resto, mostrando nuevamente diferencias significativas ( $p<0,001)(3 B)$. 
mos tratamientos de inducción (6 lavados semanales) frente a mantenimiento (más de 6 lavados). La terapia de mantenimiento demostró un mayor tiempo libre de enfermedad, con una mediana de 46 meses (intervalo de confianza del $95 \%$ de 37 a 55 meses), frente al de inducción con una mediana de 28 meses (intervalo de confianza del 95\% de 20-36 meses) (Figura 4).

Para conocer las variables explicatorias que podrían influir de forma independiente en el tiempo libre de enfermedad, empleamos el modelo de riesgo proporcional de Cox. Escogimos, por su mayor concreción, el procedimiento por pasos hacia delante (Wald), incluyendo como covariantes de estudio aquellas variables que habían mostrado diferencias significativas en el estudio univariante de supervivencia (log-rank), en este caso el empleo de instilaciones (BCG vs Quimioterapia vs nada), dosis alta de BCG frente al resto de tratamientos endovesicales (nada + quimioterapia + dosis bajas de BCG vs dosis altas de $B C G$ ) y tipo de tratamiento (inducción vs mantenimiento vs nada). Ésta última (tipo de tratamiento), al tener más de 2 valores se redefinió como categórica. Finalmente, resultaron independientes para explicar mayor tiempo libre hasta la recurrencia superficial los siguientes factores: empleo de lavados endovesica-

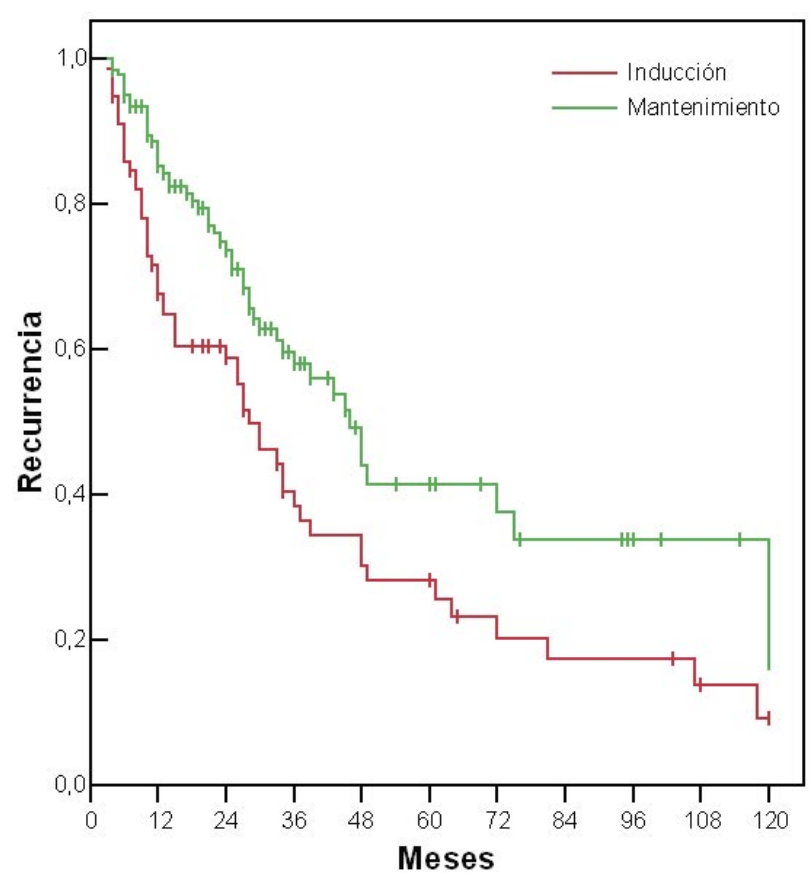

FIGURA 4. Tiempo hasta la recurrencia según se empleara terapia endovesical con protocolo de inducción (6 semanas) únicamente o con mantenimiento (más de 6 semanas), mostrando diferencias significativas (p: 0,0035). les, tratamiento con dosis altas de BCG y terapia de mantenimiento frente a inducción.

\section{DISCUSIÓN}

El empleo de terapia endovesical como tratamiento adyuvante está ampliamente descrito en la literatura. (3-9). Multitud de estudios se han publicado a este respecto, como el metaanálisis realizado por Pawinsky sobre 2535 pacientes con carcinomas superficiales primarios y recurrentes que reveló que el empleo de terapia endovesical podría reducir el riesgo de recurrencias (10), hallazgo confirmado por otros autores (11). Destacamos también el realizado sobre 1476 pacientes por la EORTC, donde se compara RTU sola frente a RTU más una instilación inmediata de un quimioterápico endovesical, concluyendo que con la resección sola se producía una recurrencia del $48,4 \%$ frente a un $36,7 \%$ en los pacientes que recibían una instilación posterior a la cirugía (12).

En nuestro estudio retrospectivo sobre 419 tumores vesicales superficiales empleamos tratamiento endovesical en el $52,3 \%$ de los pacientes, correspondiendo la mayoría a tumores T1 y de alto grado. La probabilidad de recibir tratamiento endovesical en tumores de alto grado fue significativamente mayor que la de no recibirla, si bien este riesgo se invertía en los tumores de bajo grado (en los que era más frecuente no recibir tratamiento) y resultaba equivalente en los de grado II. Además respecto al tipo de tratamiento, la probabilidad de recibir BCG era 3,5 veces superior a la de recibir quimioterapia en fumores TaIG3 y/o Tis (aislado o asociado a un tumor papilar), es decir de alto riesgo, indicaciones terapéuticas que coinciden con la mayoría de los estudios publicados $(3,9)$.

Comprobamos claramente en nuestro estudio que el empleo de instilaciones vesicales incrementó el tiempo hasta la recurrencia, con medianas de tiempo libre de enfermedad de 18 y 37 meses sin o con tratamiento, respectivamente. Además, existían diferencias significativas al emplear BCG o quimioterapia, con clara ventaja para la BCG. Según nuestros resultados, la mediana en los que fueron tratados con BCG fue de 75 meses, la de los pacientes que habían recibido quimioterapia endovesical fue de 45 meses y la de los que no recibieron tratamiento fue de 25 meses. A estas mismas conclusiones llegan otros estudios como los realizado por Bohle, demostrando una superioridad estadísticamente significativa a favor de la BCG con terapia de mantenimiento para la prevención de la progresión y recurrencia frente a la MMC $(13,14)$. 
Parece clara por lo tanto la indicación de tratar a estos pacientes con terapias adyuvantes. Podemos decir que el empleo de instilaciones vesicales incrementa el tiempo hasta la recurrencia existiendo, además, diferencias significativas al emplear BCG o quimioterapia con diferentes drogas (fundamentalmente $M M C$ ), con diferencias en las medianas de tiempo libre de enfermedad de hasta 30 meses a favor de la BCG, resultados coincidentes con varios trabajos como el meta-análisis realizado por Shelley sobre 6 estudios que incluían 1527 pacientes (15).

En cuanto al tiempo de administración así como la dosis de BCG han sido y todavía son objeto de numerosos estudios. Respecto al programa de administración se plantean dos posibilidades principalmente, un tratamiento de inducción, de 6 instilaciones semanales, o un programa de mantenimiento durante 2 a 3 años. Para la MMC la dosis por instilación es de 20 a $40 \mathrm{mg}$ disueltos en 20 a $40 \mathrm{ml}$ de agua destilada, instilándose bien en el postoperatorio inmediato o una vez por semana, durante 4 a 8 semanas con mantenimiento posterior. $(16,17)$.

El tiempo de tratamiento es, un factor implicado en la posible recurrencia del tumor, de forma que a mayor tiempo de tratamiento mayor intervalo libre de enfermedad, según nuestros resultados. La terapia de mantenimiento demostró un mayor tiempo libre de enfermedad, con una mediana de 46 meses, frente al de inducción con una mediana de 28 meses. A esta conclusión se llega igualmente en distintos estudios, como el publicado por Kolodziej y cols sobre 155 pacientes con tumores de alto riesgo que se randomizaron en RTU sola frente a RTU mas terapia de mantenimiento con BCG, objetivando progresiones en el $23 \%$ frente a el $8 \%$ de los pacientes (18).

Igualmente el análisis realizado por Griffiths y cols sobre 135 pacientes con carcinoma in situ divididos en grupos de tratamiento de inducción frente a mantenimiento, se llega también a la conclusión de que la terapia de mantenimiento con BCG ofrece los mejores resultados en cuanto a recurrencia y progresión de los tumores de alto riesgo (19). Andius y cols., sobre 236 pacientes con tumor papilar superficial, concluyen que pacientes con una primera cistoscopia negativa y terapia de mantenimiento con BCG tendrán un mayor tiempo hasta la recurrencia que aquellos tratados solo con un curso de inducción, aunque, según estos autores, no parece necesario incluir en terapia de mantenimiento a aquellos pacientes con una primera cistoscopia negativa que hallan sido $\mathrm{TaG} 1-2$, puesto que la tasa de progresión es muy baja (20). De igual forma Lamm objetiva una mejoría de la supervivencia a los 4 años del $86 \%$ con inducción frente al $92 \%$ con mantenimiento (21).

El empleo de dosis altas ha sido un tema controvertido. El grupo CUETO ha puesto en marcha varios protocolos como el 90008, donde se ha comparado la dosis reducida de $27 \mathrm{mg}$ frente a la estándar de $81 \mathrm{mg}$ en tumores de riesgo intermedio y alto, encontrando diferencias estadísticamente significativas a 40,5 meses de seguimiento en la tasa de recidiva de los tumores múltiples, siendo de $22,7 \%$ en el brazo de los $81 \mathrm{mg}$ frente a un $40,8 \%$ en el brazo de $27 \mathrm{mg}$. También se objetivan diferencias en la tasa de progresión de Tis o primarios asociados a Tis, siendo del $11,9 \%$ en el brazo de $81 \mathrm{mg}$ frente al $30,3 \%$ en el brazo de $27 \mathrm{mg}$ (109).

En el año 2002, publican nuevos resultados comparando la dosis reducida $(27 \mathrm{mg})$ frente a la dosis estandar de $81 \mathrm{mg}$, determinando que la dosis reducida tiene similares resultados para recurrencia y progresión pero con una menor toxicidad, aunque recomiendan continuar con la dosis estándar de 81 $\mathrm{mg}$ en los pacientes con tumores de alto riesgo (22).

Recientemente, en el año 2005 se han publicado los últimos resultados concluyendo que la dosis de $27 \mathrm{mg}$ es tan efectiva como la dosis de $81 \mathrm{mg}$ en la prevención de la progresión en pacientes de alto riesgo(23).

Nosotros hemos observado un tiempo libre de enfermedad mayor en los pacientes que habían recibido dosis altas de BCG, frente a los que recibieron dosis bajas de BCG y a los que recibieron quimioterapia. Encontramos que el empleo de altas dosis de BCG produjo un tiempo mayor hasta la recurrencia frente a dosis bajas o el empleo de quimioterapia.

En definitiva, aunque no hemos realizado un estudio aleatorizado y prospectivo, la revisión de nuestra serie sugiere que la terapia de mantenimiento con BCG sería el tratamiento mas adecuado en determinados tumores, siendo estos resultados coincidentes con los de otros estudios como los del SWOG, que recomienda la terapia de mantenimiento para reducir riesgo de recurrencia y progresión en carcinomas in situ y Ta o $\mathrm{Tl}$ recurrentes (24).

Finalmente, resultaron independientes para explicar mayor tiempo libre hasta la recurrencia superficial los siguientes factores: empleo de lavados endovesicales, tratamiento con dosis altas de BCG y terapia de mantenimiento frente a inducción. 


\section{CONCLUSIONES}

Hemos llegado a las siguientes conclusiones:

1. La frecuencia de empleo de tratamiento endovesical se incrementó en nuestro estudio de acuerdo al grado de malignidad. Fue significativamente más alta en pacientes con tumores de alto grado o presencia de Tis, en los que se usó fundamentalmente BCG.

2. La terapia fue poco empleada en los tumores de bajo grado, y en los tumores de grado intermedio la probabilidad de no seguir tratamiento endovesical fue similar a la de emplearla, estando basada preferentemente en quimioterapia con Mitomicina C.

3. El porcentaje de pacientes que recibió tratamiento intravesical de mantenimiento fue estadísticamente superior en caso de multiplicidad, pero no se relacionó con el estadio, grado ni con el tamaño tumoral.

4. El empleo de instilaciones vesicales incrementó de forma independiente el tiempo hasta la recidiva. Se demostró que la recurrencia se produjo significativamente más tarde cuando se habían empleado dosis altas de BCG y terapia de mantenimiento.

\section{BIBLIOGRAFIA y LECTURAS RECOMENDADAS (*lectura de interés $y^{* *}$ lectura fundamental)}

*1. LAGUNA, M.P.; VICENTE, J.: "Cirugía endoscópica de la vejiga. Tratado de endourología". Pulso Ediciones. Capitulo 4:236. 1996.

*2. ANDIUS, P.; DAMM, O.; HOLMANG, S.: "Prognostic factors in patients with carcinoma in situ treated with intravesical bacille CalmetteGuerin”. Scand. J. Urol. Nephrol.,38:285, 2004.

3. TAKASHI, M.; WAKAI, K.; HATTORI, T. y cols.: "Multivariate evaluation of factors affecting recurrence, progression, and survival in patients with superficial bladder cancer treated with intravesical bacillus Calmette-Guerin (Tokyo 172 strain) therapy: significance of concomitant carcinoma in situ". Int. Urol. Nephrol.;33:41, 2002.

4. LAMM, D.L.: "Efficacy and safety of bacille Calmette-Guerin immunotherapy in superficial bladder cancer". Clin. Infect. Dis.;31:86, 2000.

5. BRAKE, M.; LOERTZER, H.; HORSCH, R. y cols.: "Long-term results of intravesical bacillus Calmette-Guerin therapy for stage $\mathrm{T} 1$ superficial bladder cancer”. Urology. ;55:673,2000.

**6. OOSTERLINCK, W.: "Guidelines on diagnosis and treatment of superficial bladder cancer". Minerva Urol. Nefrol.;56:65, 2004.
*7. LAMM, D.L.; BLUMENSTEIN, B.A.; CRISSMAN, J.D. y cols.: "Maintenance bacillus Calmette-Guerin immunotherapy for recurrent TA, $\mathrm{T} 1$ and carcinoma in situ transitional cell carcinoma of the bladder: a randomized Southwest Oncology Group Study”. J. Urol. ; 163:1124, 2000.

8. STARTSEV, V.; POULINE, I.: "Adjuvant therapy in different risk-groups of patients with superficial bladder cancer”. Arch. Ital. Urol. Androl. ;77:93, 2005.

9. PEYROMAURE, M., GUERIN, F.; AMSELLEM-OUAZANA, D. y cols.: "Intravesical bacillus Calmette-Guerin therapy for stage $\mathrm{T} 1$ grade 3 transitional cell carcinoma of the bladder: recurrence, progression and survival in a study of 57 patients". J. Urol. ;169:2110, 2003.

10. PAWINSKY, A., SYLVESTER, R., KURTH, K.H. y cols.: "A combined Analisys of European Organization for Research and Treatment of Cancer, and Medical Research Council randomized clinical trials for the prophylactic treatment of stage TaT1 bladder cancer. European Organization for Research and Treatment of Cancer Genitourynary Tract Cancer Cooperative Group and the Medical Research Council Working Party on Superficial Bladder Cancer”. J. Urol ;156:1934. 1996.

11. HISATAKI, T.; MIYAO, N.; NASUMORI, N. y cols.: "Risk factors for multiple intravesical recurrences of superficial bladder cancer". Urology ; 58: 935, 2001.

*12. SYLVESTER, R.J.; OOSTERLINCK, W.; VAN DER MEIJDEN, A.P.: "A single immediate postoperative instillation of chemotherapy decreases the risk of recurrence in patients with stage $\mathrm{Ta}$ T1 bladder cancer: a meta-analysis of publishedresults of randomised clinical trials". J. Urol. ; 171:2186, 2004.

**13. BOHLE, A.; BOCK, P.R.: "Intravesical bacille Calmette-Guerin versus mitomycin $\mathrm{C}$ in superficial bladder cancer: formal meta-analysis of comparative studies on tumor progression". Urology ;63:682, 2004.

14. BOHLE, A.; JOCHAM, D.; BOCK, P.R.: "Intravesical bacillus Calmette-Guerin versus mitomycin $\mathrm{C}$ for superficial bladder cancer: a formal meta-analysis of comparative studies on recurrence and toxicity". J. Urol. ;169:90, 2003.

15. SHELLEY, M.D.; WILT, T.J.; COURT, J. y cols.: "Intravesical bacillus Calmette-Guerin is superior to mitomycin $\mathrm{C}$ in reducing tumour recurrence in high-risk superficial bladder cancer: a meta-analysis of randomized trials". BJU Int.;93:485, 2004.

16. CLARKE, N.S.; BASU, S.; PRESCOTT, S. y cols: "Chemo-prevention in superficial bladder cancer using mitomycin C: a survey of the practice patterns of British urologists”. BJU Int.;97:716, 2006. 
17. MOSTAFID, A.H.; RAJKUMAR, R.G.; STEWART, A.B. y cols.: "Immediate administration of intravesical mitomycin $\mathrm{C}$ after tumour resection for superficial bladder cancer". BJU Int.;97:509, 2006.

18. KOLODZIEJ, A.; DEMBOWSKI, J.; ZDOROJOWY, R.: "Treatment of high-risk superficial bladder cancer with maintenance bacilli Calmette-Guerin therapy: preliminary results". BJU Int;89:620, 2002.

19. GRIFFITHS, T.R.; CHARLTON, M.; NEAL, D.E. y cols.: "Treatment of carcinoma in situ with intravesical bacillus Calmette-Guerin without maintenance. J. Urol;167:2408, 2002.

**20. ANDIUS, P.; HOLMANG, S.: "Bacillus Calmette-Guerin therapy in stage Ta/T1 bladder cancer: prognostic factors for time to recurrence and progression”. BJU Int; 93:980, 2004.

*21. LAMM, D.L.: "Preventing progression and improving survival with BCG maintenance. Eur Urol.;37:9, 2000.

22. MARTINEZ-PINEIRO, J.A.; FLORES, N.; ISORNA, S. y cols.: "For CUETO (Club Urológico Español de Tratamiento Oncológico).: Longterm follow-up of a randomized prospective trial comparing a standard $81 \mathrm{mg}$ dose of intravesical bacille Calmette-Guerin with a reduced dose of $27 \mathrm{mg}$ in superficial bladder cancer". BJU Int ;89:671, 2002.

*23. MARTINEZ-PINEIRO, J.A.; MARTINEZ-PINEIRO, L.; SOLSONA, E. y cols.: "Club Urologico Espanol de Tratamiento Oncologico (CUETO) Has a 3-fold decreased dose of bacillus CalmetteGuerin the same efficacy against recurrences and progression of T1G3 and Tis bladder tumors than the standard dose? Results of a prospective randomized trial". J. Urol.;174:1242,2005.

24. PEYROMAURE, M.; ZERBIB, M.: "Review of the value of BCG maintenance therapy in superficial bladder tumours”. Prog. Urol. ;14:105, 2004. 